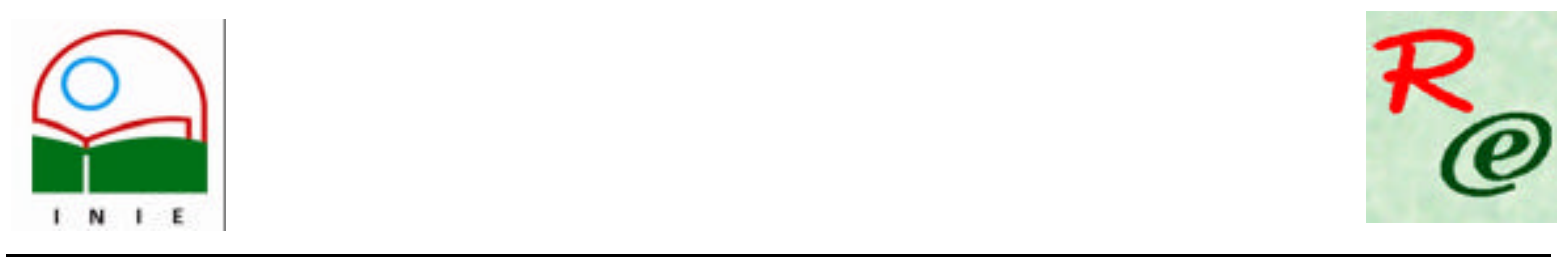

Actualidades Investigativas en Educación

Revista Electrónica publicada por el

Instituto de Investigación en Educación

Universidad de Costa Rica

ISSN 1409-4703

http://revista.inie.ucr.ac.cr

COSTA RICA

\title{
PROPUESTA DE REESTRUCTURACIÓN DEL PROGRAMA DE FÍSICA Y QUÍMICA PARA ENSEÑANZA MEDIA
}

PROPOSAL FOR THE RESTRUCTURING OF THE PHYSICS AND CHEMISTRY STUDY

PROGRAM IN SECONDARY EDUCATION

Volumen 9, Número 2

pp. 1-32

Este número se publicó el 30 de agosto 2009

Leonardo Herrera Vargas

Yeudin Artavia Herrera

La revista está indexada en los directorios:

LATINDEX, REDALYC, IRESIE, CLASE, DIALNET, DOAJ, E-REVIST@S,

La revista está incluida en los sitios:

REDIE, RINACE, OEI, MAESTROTECA, PREAL, HUASCARAN, CLASCO 


\title{
PROPUESTA DE REESTRUCTURACIÓN DEL PROGRAMA DE FÍSICA Y QUÍMICA PARA ENSEÑANZA MEDIA \\ PROPOSAL FOR THE RESTRUCTURING OF THE PHYSICS AND CHEMISTRY STUDY PROGRAM IN SECONDARY EDUCATION
}

\begin{abstract}
Leonardo Herrera Vargas ${ }^{1}$
Yeudin Artavia Herrera ${ }^{2}$

Resumen: Este artículo presenta una propuesta de reestructuración del programa de Física y Química para la enseñanza media, la cual puede ser consultada en la tesis titulada "Evolución de los programas de enseñanza media en el área de Física y de Química propuestos por el Ministerio de Educación Pública-Costa Rica, para el ciclo diversificado. Período 1980-2005". En la propuesta para el programa de Física se realizó una comparación entre los contenidos del programa de ciencias sétimo año y el programa de Física de décimo y undécimo año. Para el caso de Química la comparación se realizó entre los contenidos del programa de ciencias octavo año y el de Química de décimo y undécimo año. Dicha propuesta es producto de la triangulación que se llevó a cabo entre la revisión de los programas de enseñanza, las entrevistas realizadas a los asesores y profesores en ejercicio durante el periodo de estudio y el análisis de contenido.

Palabras claves: ENSEÑANZA DE LA FÍSICA. ENSEÑANZA DE LA QUÍMICA. MODIFICACIONES CURRICULARES. PROGRAMAS DE ESTUDIO EN COSTA RICA

Abstract: This article presents a proposal for restructuring the program in Physics and Chemistry for secondary education, which can be found in the thesis entitled "Evolution of secondary education programs in the area of Physics and Chemistry proposed by the Ministry of Public Education-Costa Rica for the diversified cycle. Period 1980-2005. ". The proposal for the program of Physics a comparison was made between the contents of the science program for seventh grade and the program of physics for tenth and eleventh grades. $\boldsymbol{h}$ the case of Chemistry a comparison was made between the content of the science program for the eighth grade and the program of Chemistry for the tenth and eleventh grades. The proposal is the result of triangulation, which took place between the revision of educational programs, interviews with counselors and in service teachers during the study and analysis of content.
\end{abstract}

Key words: PHYSICS TEACHING, CHEMISTRY TEACHING. CURRICULAR MODIFICATIONS. STUDY PROGRAMS, SECONDARY EDUCATION IN COSTA RICAN

\footnotetext{
Licenciado en la Enseñanza de la Física, Universidad de Costa Rica. Actualmente labora para la Escuela de Física de la UCR y Planetario de la Ciudad de San José de la UCR.

Dirección electrónica: leonardo.herrera@ucr.ac.cr

${ }^{2}$ Licenciado en la Enseñanza de la Química, Universidad de Costa Rica. Actualmente trabaja para el Ministerio de Educación Pública, CINDEA Alberto Manuel Brenes.
}

Dirección electrónica: yartavia82@gmail.com

Artículo recibido: 28 de mayo, 2009

Aprobado: 24 de agosto, 2009 


\section{Introducción}

La sociedad actual ha logrado un nivel de vida relativamente elevado y desea mantenerlo y aun mejorarlo. La seguridad nacional de cada país, la calidad de vida, las condiciones sanitarias y algunas otras áreas que afectan la sociedad se han obtenido por medio del desarrollo de la ciencia. Ésta se ha utilizado para crear terribles armas destructivas, para construir máquinas y desarrollar procesos que perfeccionan los bienes y servicios, y para comprender la índole de las enfermedades y el modo de controlarlas. En otras palabras, el desarrollo de la ciencia y la tecnología ha apoyado la calidad de vida pero también la ha destruido. La ciencia desempeña un papel dinámico en el progreso que el ser humano ha logrado. Es indispensable, sin embargo, que la conquista científica de lo desconocido y la aplicación técnica de lo aprendido sean continuas, para conservar lo que ya hemos logrado. (Darrell, 1970).

La Física juega un papel especial en el proceso de la enseñanza científica, pues muchos de los conceptos que son básicos para las otras disciplinas provienen de la Física. En consecuencia, ciertos conocimientos de esta ciencia deben ser introducidos en el proceso de enseñanza y aprendizaje tan tempranamente como sea posible, de manera que pueda disponerse de los elementos que son fundamentales para la construcción de nuevos conceptos en las otras disciplinas del plan de estudio. (UNICEF, 1970, p.16)

Para la enseñanza de la Química se deben reconocer enfoques que actualmente se consideran en las estructuras y procesos químicos; como es el caso de que todo estudiante debe ser enfrentado ante situaciones que tienen importancia y significado para su vida, junto con temas de importancia práctica (UNICEF, 1970, p. 17). Es por ello que la Química es considerada una ciencia práctica, en donde el educando adquiere el conocimiento en la medida que experimenta, ya sea en el campo del laboratorio o en situaciones de la vida cotidiana, de manera que se logre un aprendizaje significativo e integral afín con otras áreas del conocimiento.

La enseñanza de la Física y la Química, y la educación en general, entendida ésta como la actividad mediante la cual se produce y distribuye el conocimiento, asume en la actualidad coyuntural una importancia inédita, no sólo desde el punto de vista político-social, sino Volumen 9, Número 2, Año 2009, ISSN 1409-4703 
también desde el punto de vista de los contenidos de la socialización y su impacto en la inclusión o exclusión de amplios sectores de la población (Gurdián, 1999).

La educación adquiere importancia en el momento que es vista como un proceso continuo que ofrece al individuo la posibilidad de construir conocimientos y la oportunidad de desarrollar habilidades y destrezas que le permiten tomar conciencia de sí mismo y de su entorno y de esta forma cumplir con su rol dentro de la sociedad. Además, la educación es vista como una herramienta de desarrollo individual y de grupo, un movilizador social capaz de cerrar las brechas entre las diferentes clases sociales (Gurdián, 1999).

Los planes de estudio en el sistema educativo se proponen como los medios para plasmar los ideales y fines de la educación en un diseño curricular que contemple los elementos didácticos y pedagógicos necesarios y adecuados para la población estudiantil. Tal es el caso de los programas de estudio de Física y Química, los cuales ofrecen la posibilidad de organizar, ejecutar y evaluar la información y el conocimiento de forma integral en estas disciplinas.

Este artículo es producto de un trabajo que nace de la inquietud por conocer las modificaciones que han sufrido los programas de estudio de secundaria, en las áreas de Física y Química. Se parte del supuesto de que los programas son intentos por ofrecer espacios educativos que contribuyan significativamente como movilizadores sociales capaces de cerrar las brechas entre los diversos sectores sociales. Para este análisis se ha escogido el periodo completo de la política educativa de 1980 a 1994 y la actual política educativa hacia el siglo XXI de 1995 al 2005. Tomando en cuenta la vigencia de las dos políticas educativas mencionadas, se han seleccionado los programas de estudio del ciclo diversificado de Física y Química del periodo de 1980 al 2005. El propósito fundamental de estudio fue conocer la evolución que han sufrido los diferentes temas dentro de los programas de estudio, en el periodo mencionado. La idea es determinar los criterios que sirvieron para decidir los cambios que requerían dichos programas y así analizar los efectos que estos cambios provocaron en el proceso de enseñanza y aprendizaje de la Física y la Química. 


\subsection{Planteamiento del problema y objetivos}

Para llevar a cabo el estudio se planteó la siguiente pregunta de investigación:

¿En qué sentido evolucionaron los programas de estudio propuestos por el MEP en la enseñanza de la Física y la Química en el ciclo diversificado en el periodo de 1980-2005?

A partir de esta pregunta se propusieron los siguientes objetivos.

\section{OBJETIVO GENERAL:}

Analizar la evolución de los programas de estudio propuestos por el MEP en la enseñanza de la Física y la Química para la educación diversificada en el periodo de 1980-2005.

\section{OBJETIVOS ESPECÍFICOS:}

$\checkmark \quad$ Identificar las unidades de los programas del ciclo diversificado en Física y en Química y la lista de contenidos temáticos que se presentan en las unidades de dichos programas.

$\checkmark$ Obtener la distribución de contenidos por nivel, en el periodo de estudio.

$\checkmark \quad$ Identificar los temas que han sufrido modificaciones durante el período de estudio.

$\checkmark$ Determinar el formato de presentación de los programas de estudio que cesarrolla el MEP.

$\checkmark$ Conocer las razones de cambio en las lecciones para la enseñanza de la Física y la Química en décimo y undécimo año, en el periodo de estudio escogido.

$\checkmark \quad$ Identificar los criterios que se consideraron para decidir los cambios de los programas de estudio de Física y Química.

\section{Marco teórico}

A continuación se presenta un referencial teórico sobre el que se fundamentó la investigación, cuyo producto es este artículo. 


\subsection{Política educativa para 1980}

La Política Educativa de 1980 se inspira en los principios gubernamentales de la Doctrina Social Cristiana, así como en los postulados y en la dinámica de la democracia.

Esta Política se fundamenta en la valoración de la dignidad humana, la consecuencia del bien común, el desarrollo integral del costarricense y de la comunidad nacional, la participación popular y la justicia social, siendo los pilares fundamentales de nuestra vida institucional y serán también inspiradores y orientadores del sistema educativo nacional. (MEP 1980)

La base filosófica de la acción educativa se fundamenta en la promoción del ser humano, concebida como integradora de los aspectos materiales, espirituales y sociales.

La educación concibe al ser humano como ser creativo y le facilita su plena realización, junto con la adquisición de una alta sensibilidad social que lo haga humanizar cada una de sus propias conquistas (MEP 1980).

\subsection{Política educativa hacia el siglo XXI}

Durante la transición del siglo $\mathrm{XX}$ al siglo $\mathrm{XXI}$ se presenta un cambio de paradigma, un cambio de criterios fundamentales, una nueva manera de ver el mundo y la realidad educativa que nos rodea. Por lo que en el año de 1995 entra en vigencia la nueva y actual política educativa llamada "Política educativa hacia el siglo XXl".

La política educativa debe abordar, de forma consistente tanto los conocimientos, como los procesos de construcción y reconstrucción de los mismos, y la reflexión acerca de las consecuencias desde el punto de vista de los valores.

La Política Educativa hacia el siglo XXI se ins pira en el cambio de paradigma, al acercarse el siglo XXI que se caracteriza por una concepción de "transdisciplinariedad", en donde las disciplinas de naturaleza científica tradicional y las ciencias sociales y humanas se interconectan y se afectan mutuamente.

Toda Política Educativa, con miras a trascender periodos gubernamentales, debe establecerse sólidamente en el Marco Jurídico que rige a la Educación y en el marco político, Volumen 9, Número 2, Año 2009, ISSN 1409-4703 
económico y social de transición hacia el siglo XXI. La era de la sostenibilidad en cuatro áreas define junto con cuatro retos, el contexto en el que junto con el Marco Jurídico se enmarca la Política Educativa. Las cuatro áreas o ejes de desarrollo sostenible son: la sostenibilidad ambiental; la sostenibilidad del recurso humano; la sostenibilidad social y política y la sostenibilidad económica y productiva. (MEP, 1994).

La Política Educativa, amparada al Marco Jurídico vigente, propicia la búsqueda de un costarricense del siglo XXI que, consciente de las implicaciones éticas del desarrollo, sea una persona con rica vida espiritual, digna, libre y justa; ciudadano formado para el ejercicio participativo de la democracia, con identidad nacional; productor para sí mismo y para el país desde su condición de trabajador; solidario por experimentar como propias las necesidades de los demás, y capaz de comunicarse con el mundo de manera inteligente de tal manera que, a partir de la valoración de lo que lo identifica como costarricense, tome las decisiones que lo relacionen con otras culturas desde un punto de vista de pensador independiente, flexible y crítico, teniendo por guía los derechos y deberes humanos.

\subsection{Concepto de ciencia y tecnología}

La ciencia, según Bunge (1984), debe concebirse como un sistema conceptual, compuesto de subsistemas, que son las ciencias especiales y las interdisciplinas tales como la biofísica y la psicobiología.

La ciencia y la tecnología han sido siempre parte integral de la historia de la humanidad, contribuyendo con grandes conocimientos e inventos, los cuales han repercutido y formado el camino hacia nuevos estilos de vida de la sociedad.

\subsection{El impacto social de la ciencia}

El hablar de la ciencia y de su impacto social es un tema que no se excluye de la cultura, la cual según Taylor "es ese todo complejo que incluye conocimientos, creencias, arte, moral, leyes, costumbres y cualesquiera otras capacidades y hábitos adquiridos por el hombre como miembro de una sociedad" (Medina y otros, 2000), por lo tanto "la ciencia no puede dejar de ser cultura, ni de ser parte de la riqueza humanística del hombre". (CONICIT, 1978). 
Las culturas evolucionan en la medida en que su desarrollo social, político, económico, educativo, científico y tecnológico experimenta transformaciones significativas, como respuesta a los cambios y variaciones que se dan en el ambiente, desde el punto de vista natural y social.

Esto se hacer notar en las innovaciones científicas y tecnológicas, tal como lo menciona Medina, al decir:

Mirando hacia atrás desde el umbral del siglo $X X I$, se hace evidente que las innovaciones tecnocientíficas han sido los factores fundamentales que han configurado las culturas propias. Han modelado decisivamente el conjunto de las formas de vida, los entornos tanto materiales como interpretativos y valorativos, las cosmovisiones, los modos de organización social, económica y política junto con el medio ambiente característicos de esa época. (Medina y otros, 2000)

A lo largo de la historia el ser humano ha ido creando e innovando instrumentos y aparatos, ya sea por necesidad o simplemente por entretenimiento, lo cierto es que la funcionalidad de cada uno de ellos le ha permitido a la humanidad crecer científica y tecnológicamente, de manera que le facilita al ser humano su estilo de vida.

Durante el período griego, si bien el impacto de la ciencia sobre la sociedad por lo general se reconoce, no se presenta aquí un impacto definido de la sociedad en la ciencia, pero es indudablemente cierto que si la actividad manual mo hubiera sido despreciada en este período, los griegos hubieran realizado mucho más avance científico.

La ciencia y la tecnología han jugado un papel fundamental en la evolución social del ser humano y como tal en su formación intelectual y laboral. Es por esto que a la educación le compete contribuir con ello, facilitando una instrucción de carácter científico, orientada hacia una enseñanza de las ciencias de manera más integral y sana, para formar personas con mayor capacidad de criterio, análisis y comprensión, que sepan resolver los problemas de la sociedad y dar soluciones adecuadas según sus necesidades. 


\subsection{Importancia de la enseñanza de las ciencias}

Durante los últimos años, el progreso se presenta tan rápido que los procesos o técnicas nuevas, fueron considerados descubrimientos revolucionarios hace poco tiempo. Estos descubrimientos, posiblemente tengan hoy un valor discutible y resulten mañana anticuados. Una nueva arma nos promete seguridad si está en nuestras manos; pero en las del enem igo nos pone frente a los complejos problemas de la defensa. La magnitud y variedad de máquinas que nos permiten utilizar los recursos energéticos a velocidades astronómicas también determinan que el descubrimiento de nuevos recursos energéticos sea un problema de primerísimo orden para que podamos sobrevivir. Las drogas maravillosas que nos protegen de un microbio mortal pueden dar lugar a otro mutante, del que ninguna droga maravillosa pueda ya protegernos. Pero todos estos conocimientos científicos es necesario transmitirlos a las nuevas generaciones, de ahí la importancia de la enseñanza de las ciencias.

La adquisición del conocimiento científico, lejos de ser un producto espontáneo y natural de nuestra interacción con el mundo de los objetos, es una laboriosa construcción social, o mejor aún re-construcción, que sólo podrá alcanzarse mediante una enseñanza eficaz que sepa afrontar las dificultades que ese aprendizaje plantea.

Para que el conocimiento sirva de base a la acción humana, para que el conocimiento científico y la reflexión filosófica sean base de la transformación del mundo, deben dar un paso difícil pero indispensable: tienen que ser comprensibles para la gente pero, más aún, tienen que ser capaces de convertirse en algo más que conocimiento: tienen que ser creíbles para la gente, tienen que volverse parte de nuestro sistema de creencias, tienen que ser creídos, no simplemente sabidos. En otras palabras, tienen que pasar a ser parte de nuestra cultura. Por esas razones, la labor de la educación científica es lograr que los alumnos construyan en las aulas actitudes, procedimientos y conceptos que por sí mismos no lograrían elaborar en contextos cotidianos y que, siempre que estos conocimientos sean funcionales, los trasfieran a nuevos contextos y situaciones. De esta forma, el currículo de las ciencias, desarrollado a través de las actividades de aprendizaje y enseñanza, debe servir como una auténtica ayuda pedagógica, una vía para que el alumno acceda a formas de conocimiento que por sí mismas le serían ajenas o al menos muy distantes. 


\subsection{Las escuelas educan a nuestros científicos}

El desarrollo de la ciencia es obra de las personas. Los grandes descubrimientos científicos son fruto de los esfuerzos humanos para interpretar los hallazgos de sus investigaciones. Las aplicaciones técnicas de esos descubrimientos para ampliar el bienestar de la comunidad, también son obra del ser humano. Toda la ciencia es creación humana, y su futuro, en los dominios del progreso teórico y técnico, dependerá de la calidad y número de nuestros futuros científicos.

Nuestra necesidad de mano de obra con preparación científica es mayor que en cualquier otro período histórico. Pero, en proporción, son menos los estudiantes universitarios que hoy se preparan para carreras científicas. En los Estados Unidos muchos ciudadanos consideran esta situación como uno de los problemas más graves del país. Este hecho puede estar relacionado, con la situación que impera, por ejemplo, en el sistema educativo de España, ya que un estudio sobre la forma en que los profesores de ciencias de secundaria conciben la evaluación en ese país demostró que casi el $90 \%$ de los profesores de Física y Química están convencidos de que en su materia una evaluación adecuada es aquella que "suspende" a la mitad de los alumnos. Si la mayoría aprueba tienden a creer que esa evaluación ha estado mal diseñada. Esto es muy crítico porque puede ser el motivo de que cada vez más estudiantes pierdan el interés o la motivación y desistan de optar por carreras científicas a nivel universitario. Esta misma situación puede ser también la realidad que se está viviendo en Costa Rica y quizás en el resto del mundo.

Por eso, hay que buscar nuevas metas educativas para la educación, dirigidas más a desarrollar en los alumnos capacidades que les permiten afrontar los cambios culturales que se están produciendo no solo en la vida social, sino sobre todo en los perfiles profesionales y laborales y en la propia organización y distribución social del conocimiento.

\subsection{Las escuelas educan a los futuros ciudadanos de la sociedad tecnológica}

En una democracia los ciudadanos deben tomar muchas decisiones. Entre ellas, las que conciernen al apoyo financiero de la investigación científica, ya sea por parte de corporaciones privadas o por organismos gubernamentales, que dependen en último término del público, que también decide como se deben manejar los recursos naturales. Los ciudadanos determinan las características de los programas sanitarios, nacionales y locales, 
al declarar en qué medida están dispuestos a contribuir y, por ende qué grado de eficacia y permanencia pueden alcanzar esos programas. Para que las decisiones que se tomen en este y otros asuntos sean inteligentes, es necesario contar con una ciudadanía provista de conciencia científica. Lograr ese nivel es responsabilidad de los centros educativos y, dentro de ellas, en especial, de la enseñanza científica. Como las instituciones educativas pertenecen al pueblo, esta es la autoridad final para decidir qué debe enseñarse y que tipo de instalaciones deberán emplear para ello.

Sin embargo, la realidad no es así, se sabe que el estado es el que tiene control de todos los bienes y servicios públicos, y el debe de educar ciudadanos para que enfrenten una sociedad tecnológicamente activa, requiere de una gran inversión con que el pueblo no cuenta, ya que es necesario suministrar a las instituciones educativas equipos tecnológicos y aulas adecuadas para desarrollar el conocimiento, las habilidades y las destrezas tecnológic as en los estudiantes. Por otro lado, se debe realizar un currículo (para el cual no es consultado el pueblo) donde se considere cuáles son los objetivos, contenidos, procedimientos, evaluación, entre otros elementos, los cuales se espera que el alumno desarrolle, para que pueda tener las herramientas necesarias para enfrentarse ante una sociedad tecnológica.

\subsection{Las escuelas educan para facilitar la adaptación}

Desde que son concebidos, pasando por sus primeros meses de vida y luego por la infancia, la adolescencia, la edad adulta y la vejez, los seres humanos cambian siempre, en cuanto son organismos biológicos en funcionamiento. Cambian en su modo de sentir acerca de sí mismos hacia de los demás y el modo en que los trata la sociedad. En cada etapa de su desarrollo, el individuo debe satisfacer ciertas necesidades si aspira a lograr una relación favorable consigo mismo, con sus semejantes y con su medio. La identificación de esas necesidades y el examen del papel que debe desempeñar la educación para satisfacerlas, son factores que contribuyen a definir los objetivos, contenidos y métodos de todo programa educativo. En el caso de los adolescentes, una buena selección de contenidos y métodos científicos puede contribuir a satisfacer esas necesidades.

La gente joven posee variados intereses que es posible despertar y fomentar por medio de programas científicos debidamente planeados. Cuando entran a estudios de secundaria Volumen 9, Número 2, Año 2009, ISSN 1409-4703 
muchos ya han revelado un talento especial que con la guía de un programa científico rico y estimulante los llevará luego a seguir carreras científicas. Todos los estudiantes de secundaria en general, hallan en los programas de ciencias situaciones que se relacionan con sus problemas personales y sociales, una ayuda para formarse un concepto propio del papel decisivo que desempeña la ciencia en el moderno mundo tecnológico. Los jóvenes pueden tener experiencias científicas que les crean actitudes mentales y espíritu crítico, capaces de permitirles afrontar mejor los problemas y dar a estos las soluciones más adecuadas. Tales atributos son básicos para la solución inteligente de problemas personales y sociales y, por lo mismo, para una más eficiente educación cívica. Aunque la formación de esas actitudes y capacidades puede también integrarse a un programa de estudio sociológico, la ciencia puede aportar una contribución que es única.

\subsection{Una base para elegir generalidades}

En términos de los objetivos de contenido o temas científicos, es evidente que los docentes deben enseñar tratando de hacer comprender cada vez más las generalidades científicas. Se han publicado muchas y extensas listas de generalizaciones científicas escogidas y muchas de las cuales, además se mencionan como principios científicos.

Todas las listas fueron preparadas por investigadores, según técnicas de minucioso control. Hay listas de generalidades o principios de ciencias biológicas, físicas, naturales, geología, entomología, edafología, conservación y muchas otras. Ningún docente podría enseñar ni siquiera una pequeña fracción del total. Pero el valor de esas listas reside en su carácter de fuentes autorizadas para elegir de ellas las generalizaciones que van a enseñarse. Por lo tanto, los docentes respectivos deben tener una base que les permite seleccionar las generalizaciones o principios que les parezcan más adecuados para alcanzar la meta que se hayan propuesto en la enseñanza de los cursos.

Existen por lo menos dos métodos para seleccionar y utilizar generalizaciones cuando se enseñan ciencias. Ambos se basan en puntos de vista educativos claramente definidos. Por uno de estos métodos, el docente elige aquellas generalizaciones que, a su juicio o al juicio de los expertos, son más importantes para que sus alumnos comprendan. Luego organiza su curso para enseñarles a comprender esas generalizaciones escogidas. Por el otro método, el docente considera primero los problemas personales y sociales que formarían el núcleo de Volumen 9, Número 2, Año 2009, ISSN 1409-4703 
organización del curso o plan de estudios. Luego utiliza aquellas generalizaciones que tienen utilidad directa para resolver tales problemas. La enseñanza, en cuanto se relaciona con generalizaciones que deben ser aprendidas, puede ser igualmente eficaz en ambos casos. En Costa Rica, el encargado de controlar los programas de cursos que enseñan esas ciencias es el Ministerio de Educación Publica, ente controlador del sistema educativo costarricense, cuya estructura administrativa presenta asesores por materia, lo que permite contar con un asesor nacional para cada ciencia. Esa estructura del MEP se ofrece en la siguiente temática.

\subsection{Marco referencial de la educación costarricense}

Se presenta una visión desde el punto de vista histórico, acerca de la evolución de la educación costarricense, con énfasis en la situación actual del sistema educativo nacional. En la Constitución Política de la República, se incorpora, a partir de 1949, un capítulo acerca de la educación y la cultura, que no sólo mantiene los logros del pasado sino que amplía conceptos y garantías muy importantes.

En el artículo 78 de dicha constitución se establece que:

La educación preescolar y la general básica son obligatorias. Estas y la educación diversificada en el sistema público son gratuitas y costeadas por la Nación. En la educación estatal, incluida la superior, el gasto público no será inferior al seis por ciento $(6 \%)$ anual del producto interno bruto, de acuerdo con la ley... El Estado facilitará la prosecución de estudios superiores a quienes carezcan de recursos pecuniarios. La adjudicación de las becas y los auxilios estará a cargo del Ministerio del ramo, por medio del organismo que determine la ley. ${ }^{(2)}$

Al incorporarse el artículo citado anteriormente a la constitución política se evidencia el inicio de la comprensión por parte de los políticos de la gran importancia que tiene la educación para llevar al desarrollo un país.

\subsection{Programas de estudio.}

Los programas de estudio son de suma importancia para alcanzar los ideales educativos de una nación, que no solo corresponden a fines ideológicos o filosóficos, también representan un medio que aporta conocimientos y bases para el desarrollo de nuevas habilidades, 
destrezas y actitudes, en la formación de un individuo de provecho para el progreso del país. En Costa Rica, con los programas de estudio se pretende lograr en los educandos no solo un mayor nivel cognitivo, sino también una mejor formación en valores y actitudes, orientados bajo una política humanista, racionalista y constructivista, que conduzcan al individuo hacia su desarrollo integral.

A continuación se detalla, según el MEP, lo que es un programa de estudio y sus componentes, y cuál es el enfoque u orientación que estos desempeñan en el área de la Física y de la Química.

Los PROGRAMAS DE ESTUDIO deben ser una guía relevante para el docente desde el punto de vista de la planificación de su trabajo de mediación en coherencia con los recursos didácticos, el proceso de evaluación y sus propias necesidades e intereses profesionales. Todo programa de estudio debe incluir objetivos, contenidos, experiencias y estrategias de mediación del aprendizaje y evaluación. (MEP, 1994, p.12)

Estos programas están integrados por varios componentes:

LOS OBJETIVOS deben contemplar los contenidos, los procesos cognitivos y los valores que se persiguen realizar durante el ciclo lectivo en cuestión sobre la base del ciclo lectivo anterior, con una perspectiva de crecimiento y profundización hacia el ciclo lectivo venidero.

LOS CONTENIDOS deben organizarse con base en conocimientos sólidos y rigurosos de cada disciplina; deben ser claros y autoexplicativos, consecuentes con la planificación de los objetivos.

LAS EXPERIENCIAS Y ESTRATEGIAS DE MEDIACIÓN DEL APRENDIZAJE deben contribuir al desarrollo del conocimiento y a su construcción, al desarrollo del pensamiento y a la consolidación de valores.

LA EVALUACIÓN además de ser coherente con respecto a los objetivos, debe permitir verificar y valorar su cumplimiento. (MEP, 1994, p. 12) 
Una ampliación sobre el marco teórico que fundamentó este trabajo puede ser consultado en el artículo que se publicó en el Volumen 8, Número 3 Revista Electrónica Actualidades Investigativas en Educación titulado Marco referencial para el análisis de los programas de enseñanza media en el área de Física y de Química propuestos por el Ministerio de Educación Pública Costarricense.

\section{Metodología general}

Para el desarrollo del trabajo de investigación se procedió de la siguiente manera:

$\checkmark$ Se solicitó en las oficinas del MEP los programas de Física y de Química de décimo y undécimo año del periodo 1980-2005.

$\checkmark$ Se procedió a realizar una revisión minuciosa de los programas mencionados para identificar las unidades en que se dividieron dichos programas en ese período. Igualmente se hizo con los contenidos. Se escribió una lista de los temas pertenecientes a esas unidades, así como la distribución de los contenidos por nivel.

$\checkmark$ Se identificaron los temas que han sufrido modificaciones durante el periodo mencionado, tratando de ubicar si existe algún aprendizaje interdisciplinario, al comparar con otros programas que no son parte de este estudio.

$\checkmark \quad$ Se analizaron las modificaciones del punto anterior para identificar la razón de esos cambios, tratando de ubicar si existe algún aprendizaje interdisciplinario, al comparar con otros programas (Ciencias y Biología) que no son parte de este estudio.

$\checkmark \quad$ Se determinó el número de lecciones que se impartían semanalmente, en la enseñanza de la Física y Química de décimo y undécimo año.

$\checkmark \quad$ Se entrevistó a los asesores nacionales de Física y Química que cumplieron esa función en el periodo de estudio.

$\checkmark$ Se entrevistó a docentes de alta experiencia que ejercieron su profesión en el periodo de estudio.

$\checkmark \quad$ Se describió el formato de presentación de los programas del MEP.

$\checkmark$ Análisis de la información:

$\checkmark$ Contenidos: se analizó por medio de tablas de doble entrada los contenidos y año en que se cubrieron.

$\checkmark$ Entrevistas: Se utilizó para complementar la información anterior.

$\checkmark$ Se realizó una triangulación entre: revisión de documentos, entrevistas y análisis de contenido. 


\section{Análisis de resultados}

\subsection{Triangulación de la información obtenida}

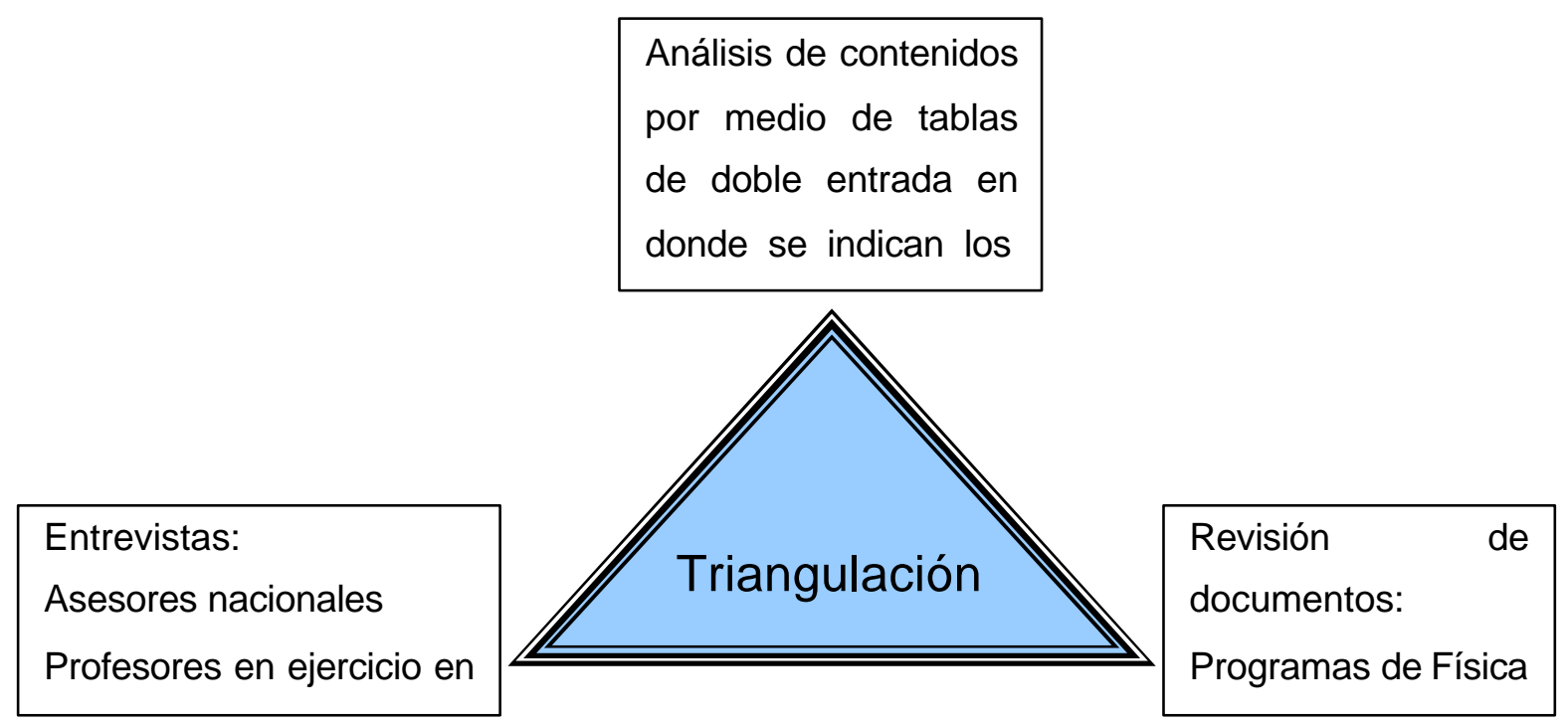

Se presenta el análisis de los resultados de Física y de Química, como producto de la triangulación que se llevó a cabo entre la revisión de los programas de enseñanza, las entrevistas realizadas a los asesores y profesores en ejercicio durante el periodo de estudio y el análisis de contenido. Aquí se hace referencia a información ubicada en cuadros que se localizan bajo los títulos de "Cuadros de Física" y "Cuadros de Química", los cuales pueden ser consultados en la tesis titulada "Evolución de los programas de enseñanza media en el área de Física y de Química propuestos por el Ministerio de Educación Pública, para el ciclo diversificado. Período 1980-2005". 
A continuación se presenta un resumen de los cuadros de Física y de Química donde se muestran algunos aspectos generales:

Cuadros de Física, décimo año

\begin{tabular}{|c|c|}
\hline \multirow[t]{2}{*}{$\begin{array}{l}\text { I Unidad: Historia inicial del universo } \\
\text { Contenidos }\end{array}$} & \multirow{5}{*}{$\begin{array}{l}\text { Esta unidad se elimino del programa por } \\
\text { las siguientes razones: } \\
\text { Falta de preparación del docente al impartir } \\
\text { este tema debido a que en su formación } \\
\text { académica no recibe ninguna preparación } \\
\text { con respecto a este tema. }\end{array}$} \\
\hline & \\
\hline & \\
\hline 2. Origen y fin de la gran explosión. & \\
\hline $\begin{array}{l}\text { 3. Comportamiento del universo desde la } \\
\text { explosión inicial y la fuerza de atracción } \\
\text { gravitacional. }\end{array}$ & \\
\hline 4. Velocidad de escape. & \multirow{4}{*}{$\begin{array}{l}\text { Ajuste del programa con respecto al } \\
\text { temario de bachillerato, al no formar parte } \\
\text { de este se elimina. } \\
\text { Debido que al docente le falta tiempo para } \\
\text { impartir todo el programa se elimina esta } \\
\text { unidad. }\end{array}$} \\
\hline 5. Formación y evolución de galaxias. & \\
\hline 6. Constitución y evolución de las estrellas. & \\
\hline $\begin{array}{l}\text { 7. Formulación de la teoría de la "Gran } \\
\text { explosión" y descripción del origen del } \\
\text { sistema solar. }\end{array}$ & \\
\hline $\begin{array}{l}\text { I Unidad: Conceptos Fundamentales de } \\
\text { Física } \\
\text { Contenidos }\end{array}$ & \multirow{9}{*}{$\begin{array}{l}\text { Dentro de la unidad de conceptos } \\
\text { fundamentales de Física, se introducen una } \\
\text { serie de contenidos que son fundamentales } \\
\text { para el estudio de contenidos posteriores, } \\
\text { con el objetivo de facilitarle la comprensión } \\
\text { al estudiante. }\end{array}$} \\
\hline$\checkmark$ Intensidad de corriente eléctrica & \\
\hline$\checkmark$ Cifras significativas. & \\
\hline $\begin{array}{l}\text { Notación científica y potencias de base } \\
\text { diez. }\end{array}$ & \\
\hline$\checkmark$ Conversiones de unidades del S.I: & \\
\hline$\checkmark \quad$ ¿Qué es la física? & \\
\hline$\checkmark$ Magnitudes básicas del SI: & \\
\hline$\checkmark$ Area y Volumen & \\
\hline$\checkmark$ Densidad & \\
\hline
\end{tabular}




\section{Unidad: Movimiento rectilíneo de los Cuerpos: Cinemática Contenidos}

a. Algunos temas del Movimiento uniforme en línea recta:

$\checkmark$ Rapidez media

$\checkmark$ Velocidad media

$\checkmark$ Velocidad instantánea

$\checkmark$ Construcción e interpretación de gráficos

$\mathrm{d}-\mathrm{t}, \quad \vec{d}-\mathrm{t}, \quad \vec{v}-\mathrm{t}, \quad \mathrm{v}-\mathrm{t}$
En año de 1991 se introduce en esta unidad algunos conceptos fundamentales para la comprensión de dicho tema al igual que la construcción e interpretación de gráficas que facilitan al estudiante un análisis visual de diferentes mediciones las cuales se recomiendan que se realicen de forma experimental.

\section{Cuadros de Física, undécimo año}

\begin{tabular}{|c|c|}
\hline $\begin{array}{l}\text { III Unidad: Optica y Ondas } \\
\text { Contenidos }\end{array}$ & Comentario \\
\hline$\checkmark \quad$ Naturaleza de la luz: & \multirow{6}{*}{$\begin{array}{l}\text { En el programa de } 1996 \text { se introduce este } \\
\text { tema para lograr una mayor comprensión } \\
\text { por parte del estudiante al estudiar los } \\
\text { diferentes comportamientos que presenta la } \\
\text { luz, en diferentes superficies y al crear } \\
\text { imágenes. } \\
\text { Se introduce el tema de descomposición de } \\
\text { la luz con el objetivo de que el estudiante } \\
\text { logre una mayor comprensión del } \\
\text { comportamiento de la luz. }\end{array}$} \\
\hline$\checkmark \quad$ Luz visible & \\
\hline$\checkmark \quad$ Fuentes de luz & \\
\hline$\checkmark$ Descomposición de la luz visible: & \\
\hline$\checkmark \quad$ El arco iris & \\
\hline $\begin{array}{l}\checkmark \quad \text { Unidad de medida en el S.I; de la ley de la } \\
\text { iluminación }\end{array}$ & \\
\hline$\checkmark \quad$ Defino aberración esférica. & $\begin{array}{l}\text { Estos temas son eliminados básicamente } \\
\text { por el reajuste del programa con el temarid } \\
\text { de bachillerato. }\end{array}$ \\
\hline$\checkmark$ Temas del movimiento ondulatorio: & \multirow{7}{*}{$\begin{array}{l}\text { Se introducen diferentes contenidos } \\
\text { característicos de las ondas en el programa } \\
\text { de 1996, con el objetivo de lograr un } \\
\text { aprendizaje más significativo por parte de } \\
\text { los estudiantes }\end{array}$} \\
\hline$\checkmark \quad$ Concepto & \\
\hline$\checkmark$ Tipos de ondas: & \\
\hline$\checkmark \quad$ Electromagnéticas & \\
\hline$\checkmark \quad$ Mecánicas: & \\
\hline$\checkmark \quad$ Transversales & \\
\hline$\checkmark \quad$ Longitudinales & \\
\hline
\end{tabular}




\section{Cuadros de Química, décimo año}

\begin{tabular}{|c|c|}
\hline \begin{tabular}{l}
\multicolumn{2}{c}{ Contenidos } \\
- Contribuciones de la Química al \\
mejoramiento de la calidad de vida.
\end{tabular} & \multirow[b]{2}{*}{$\begin{array}{l}\text { Se introdujeron a partir del programa del año } \\
1991 .\end{array}$} \\
\hline $\begin{array}{l}\text { - Impacto de las sustancias químicas en el } \\
\text { ambiente, la alimentación, la salud y el } \\
\text { desarrollo sostenible en general. }\end{array}$ & \\
\hline $\begin{array}{l}\text { Elementos esenciales en los organismos } \\
\text { vivos (oligoelementos). }\end{array}$ & \multirow[b]{2}{*}{$\begin{array}{l}\text { Este contenido se introdujo a partir del año } \\
1996 .\end{array}$} \\
\hline $\begin{array}{l}\text { - Uso y manejo de compuestos químicos. } \\
\text { - Contaminación por reacciones químicas }\end{array}$ & \\
\hline $\begin{array}{l}\text { Disoluciones: componentes, } \\
\text { clasificación, cálculos sobre } \\
\text { porcentajes peso en peso, peso en } \\
\text { volumen, volumen en volumen. } \\
\text { Molaridad. }\end{array}$ & \multirow{3}{*}{$\begin{array}{l}\text { Estos contenidos fueron eliminados del } \\
\text { programa de décimo año y trasladados para } \\
\text { undécimo año a partir del año } 1996 \text {. }\end{array}$} \\
\hline $\begin{array}{l}\text { - Solubilidad: factores de los cuales } \\
\text { depende. }\end{array}$ & \\
\hline $\begin{array}{l}\text { - Importancia de las disoluciones en la } \\
\text { naturaleza y en la vida diaria. }\end{array}$ & \\
\hline - Métodos de separación de la materia. & $\begin{array}{l}\text { Este contenido señalado fue trasladado para } \\
\text { el programa de octavo año a partir de } 1996 \text {. }\end{array}$ \\
\hline - Estados de agregación de la materia. & $\begin{array}{l}\text { Este contenido se elimino del programa de } \\
\text { décimo año pero se incluyó en el programa } \\
\text { de octavo año, a partir del año } 2005 \text {. }\end{array}$ \\
\hline $\begin{array}{l}\text { Tabla Periódica de los Elementos: } \\
\text { Desarrollo histórico. Aportes } \\
\text { hechos por Dobereiner, Newland, } \\
\text { Meyer, Mendeleiev y Moseley. }\end{array}$ & \multirow[t]{2}{*}{$\begin{array}{l}\text { Este contenido fue eliminado del programa } \\
\text { de décimo año. }\end{array}$} \\
\hline $\begin{array}{l}\text { Moléculas diatómicas, triatómicas, } \\
\text { tetratómicas, poliatómicas, etc. }\end{array}$ & \\
\hline
\end{tabular}




\section{Cuadros de Química, undécimo año.}

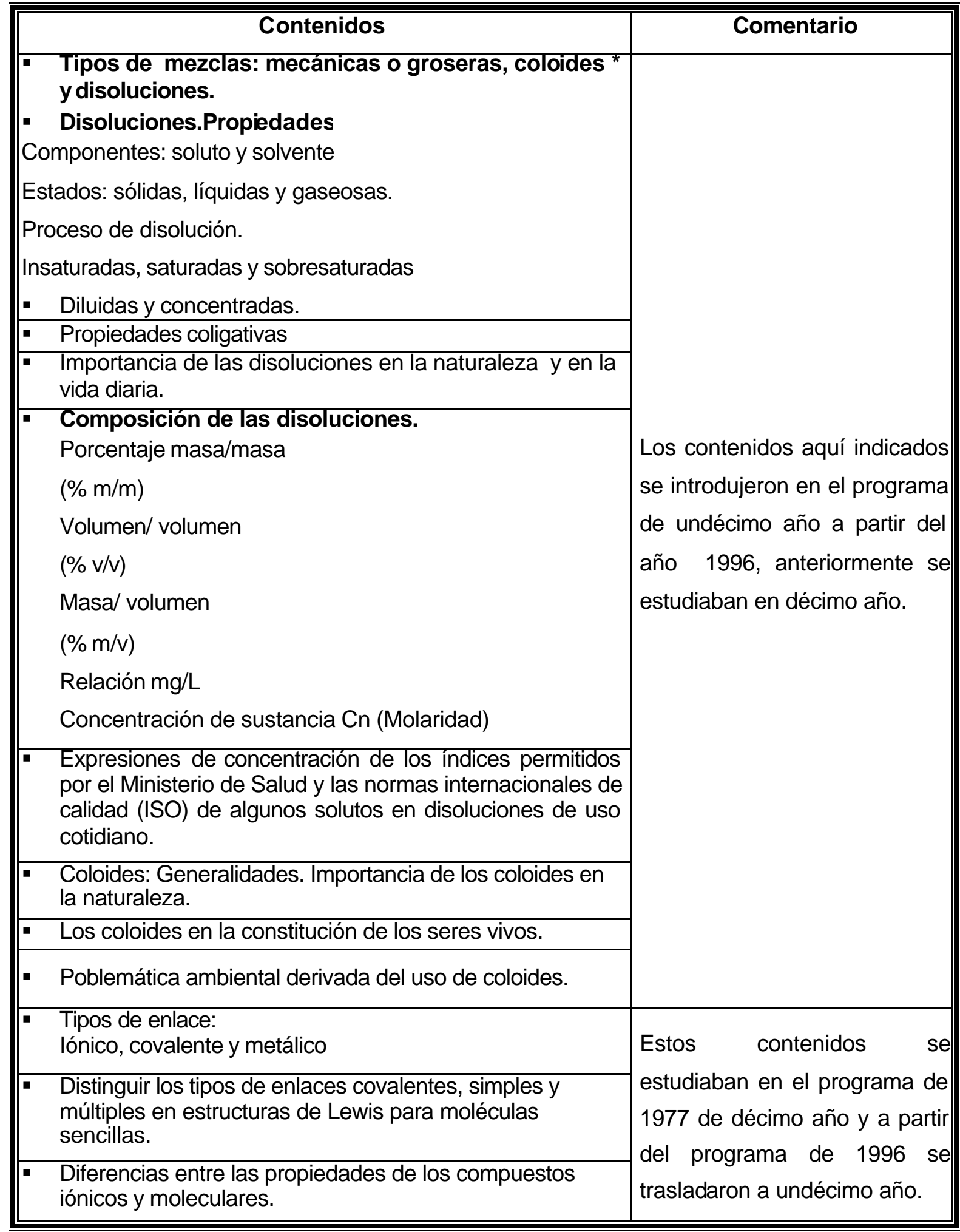


Estados de la materia. Características macroscópicas y microscópicas.

- Interacciones intermoleculares: Van der Waals y puentes de hidrógeno.

\section{Conclusiones y recomendaciones}

\subsection{Conclusiones en el área de la enseñanza de la Física}

Los programas de estudio de Física que ofrece el MEP han recibido diferentes modificaciones en el periodo de 1980 hasta el 2005. Se encuentra que sus principales modificaciones se deben a los siguientes puntos:

$\checkmark \quad$ En el ciclo de educación diversificada los programas de Física presentan temáticas que son importantes para comprender la ciencia de hoy día, pero que corresponden a conceptos que se descubrieron en épocas anteriores al siglo XIX. Si bien son importantes para comprender la ciencia de hoy, es necesario incluir temas del siglo actual en los programas.

$\checkmark \quad$ Las modificaciones que se le realizan a los programas de estudio de Física no están acordes con los avances tecnológicos, ya que no contemplan temáticas de las nuevas tecnologías ni los descubrimientos científicos de los siglos XX y XXI.

$\checkmark \quad$ Los docentes de matemática y Física no coordinan entre sí para que cuando, en el desarrollo de los programas de Física se requieran ciertos conocimientos matemáticos, los estudiantes cuenten con ellos.

$\checkmark \quad$ La eliminación de los temas de la unidad "Historia inicial de Universo" se justifica porque algunos docentes no tenían los conocimientos para desarrollarlos, según manifestaron los asesores entrevistados, quienes consideraron esta situación para proponer los cambios.

$\checkmark \quad$ De acuerdo con la opinión de los asesores, la enseñanza de la Física debe de darse en un ambiente práctico para que los estudiantes adquieran un aprendizaje significativo. 


\subsection{Conclusiones en el área de enseñanza de la Química}

Al analizar los programas de estudio de la Química propuestos por el MEP, en el período establecido, se encuentra que estos han sufrido modificaciones las cuales se deben a las siguientes razones:

$\checkmark \quad$ Los cambios que se aplicaron en el programa permiten mantener la secuencia de los conceptos.

$\checkmark \quad$ La introducción de nuevos temas se debió a que el desarrollo científico-tecnológico obligó a tener una mayor conciencia sobre la responsabilidad e importancia que conlleva el uso y manejo de sustancias Químicas, presentes en los organismos vivos, en la industria, en el hogar, en la agricultura y en otras actividades del ser humano.

$\checkmark \quad$ La eliminación de algunos temas se debió a la falta de capacitación y de preparación de los docentes, los cuales no se sentían aptos para desarrollarlos. Además se consideró que ciertos contenidos tenían que ser trasladados al nivel universitario, según la información que ofrecieron los docentes entrevistados.

$\checkmark \quad$ No hay un verdadero aporte para un aprendizaje interdisciplinario. Por ejemplo, el tema de biomoléculas (carbohidratos, lípidos y proteínas), que corresponde a la parte de química orgánica, se estudia hasta el tercer trimestre, según el programa de química de undécimo año, mientras que en el programa de biología de décimo año se estudia este tema en el primer trimestre. Esto se evidencia al revisar los programas de Química, en los cuales se encuentran conceptos que tienen relación con los de biología, programa que se revisó para ver la interdisciplinaridad. Es necesario que los profesores de Química coordinen con los profesores de biología.

$\checkmark \quad$ En la práctica el programa de Química ro estimula a que el docente identifique y relacione los conceptos químicos con su aplicación tecnológica actual, ya que muchos profesores y centros educativos no cuentan con herramientas y equipo tecnológico de actualidad, ni tampoco son capacitados por parte del MEP en esta área, estos más bien incitan hacia un interés científico.

$\checkmark \quad$ Algunos temas del programa sugieren hacer uso de laboratorios y la mayoría de los colegios no cuentan con este recurso, por lo que no se puede cumplir con todo lo que propone el programa. Según la entrevista a la asesora nacional de Química, realizada en octubre del 2007, no existe un registro real del uso que se les da a los laboratorios de Química en las instituciones de educación media. Algunos son utilizados como aulas, otros se encuentran cerrados por falta de mantenimiento y reactivos. Si bien esta Volumen 9, Número 2, Año 2009, ISSN 1409-4703 
investigación no contempló indagar la existencia de laboratorios de Química a nivel medio, la asesora nacional de Química cuenta con la información sobre ellos. Esto lleva a la conclusión de que algunos aspectos del programa no se pueden concluir tal como se proponen.

\subsection{Conclusiones Generales y recomendaciones}

Los programas de estudio de la Física y la Química que ofrece el MEP han sufrido diferentes modificaciones en el periodo de 1980 hasta el 2005. Se encuentra que sus principales modificaciones se deben a los siguientes puntos:

$\checkmark \quad$ Los docentes consultados perciben que los cambios en los programas son producto de intereses políticos, una frase muy común entre los docentes, ya que no se enteran de cómo se realizan estos cambios, pues sienten que no se realizó una previa consulta a los profesionales del tema.

$\checkmark \quad$ Durante el período de estudio, las lecciones de química eran cuatro lecciones en cada nivel y estas se redujeron a tres lecciones por semana, lo que provoca la necesidad de reducir los temas.

$\checkmark \quad$ Se realiza un reajuste de los contenidos del programa con respecto al temario de bachillerato con el objetivo de que los docentes cumplan el programa y no desarrollen el temario.

$\checkmark \quad$ Se observa que en el período de estudio de los programas, estos sufren cambios que no están acordes con el avance del desarrollo científico, ya que se quedan en conocimientos científicos que se desarrollaron antes de los años 50.

Al realizar el análisis de los programas de estudio, se proponen las siguientes recomendaciones:

$\checkmark \quad$ Antes de hacer las modificaciones de los programas, consultar a los docentes como primeros usuarios de los programas de estudio, al diseñar un nuevo programa.

$\checkmark \quad$ Hacer un cartel de alcance y secuencia para realizar una revisión de la distribución temática y de la coordinación que presentan los programas de ciencias del tercer ciclo y de la educación diversificada, para evitar repeticiones de contenidos.

$\checkmark \quad$ Diseñar programas de estudio acordes con los avances científicos y tecnológicos. 
$\checkmark \quad$ Proveer a los colegios de equipo tecnológico y de laboratorio para cumplir a cabalidad el desarrollo de los programas e incentivar en los estudiantes una mayor motivación y gusto por el aprender ciencias.

$\checkmark \quad$ Hacer uso de laboratorios virtuales, en aquellas instituciones que cuentan con el equipo de cómputo adecuado.

$\checkmark \quad$ Se propone realizar una reestructuración de los contenidos para el programa de Física y de Química de la educación diversificada.

Haciendo uso de los conocimientos construidos en esta investigación se presenta una propuesta de lo que deben ser los programas de física y de química para el ciclo diversificado.

\subsection{Propuesta de reestructuración de los contenidos de los programas de estudio de Física y de Química.}

En la propuesta de reestructuración para el programa de Física se realizó una comparación entre los contenidos del programa de ciencias sétimo año y el programa de Física de décimo y undécimo año.

Para el caso de Química la comparación se realizó entre los contenidos de los programas de ciencias octavo año y el de Química de décimo y undécimo año.

En ambos casos se identificaron, de forma separada, los contenidos que se repetían en los programas de estudios analizados. De esta manera se plantea una propuesta de reestructuración para el programa de décimo y undécimo año en donde aparezca como una única vez el contenido a desarrollar.

5.4.1 Propuesta de reestructuración para el programa de Física de 10ำ

Propuesta $10^{\circ}$ año

- ¿Qué es la Física?

- Evolución de la Física a través de la historia.

- Importancia del conocimiento físico en el desarrollo científico y tecnológico.

- Relación de la Física con otras ciencias. 
- Magnitudes básicas delS.I:

- Longitud, Tiempo, Masa, Intensidad luminosa,

- Intensidad de corriente eléctrica,

- Temperatura termodinámica, Cantidad de materia.

- Prefijos para unidades de medida.

- Cifras significativas.

- Notación científica y potencias de base diez.

- Conversiones de unidades del S.I:

- Derivadas

- Reseña histórica del concepto de movimiento.

- Cantidades vectoriales y escalares.

- Representación gráfica de vectores

- Método de componentes vectoriales

- Movimiento con aceleración constante en línea recta:

- Aceleración media e instantánea.

- Construcción e interpretación de gráficos $v-t$ y $a-t$

- Nuclear débil, fuerte

- Movimiento vertical en las inmediaciones de la superficie terrestre (caída libre):

- Movimiento vertical

- Movimiento parabólico.

- Concepto de fuerza y su unidad en el S.I.

- Energía

- Cinética

- Potencial: Gravitatoria y Elástica

- Mecánica

- Conservación de la energía mecánica en planos inclinados, movimiento vertical, péndulos, resortes, tobogán

- Potencia

- Otras energías

- Concepto

- Unidades de potencia

- Impulso y cantidad de movimiento para una partícula.

- Impulso y cantidad de movimiento para muchas partículas.

- Choques elásticos e inelásticos en una dimensión.

- Movimiento circular uniforme:

- Velocidad tangencial

- Rapidez tangencial

- Período y frecuencia

- Aceleración centrípeta

- Fuerza centrípeta

- Movimiento planetario. reseña histórica de los modelos Geocéntrico y Heliocéntrico.

- Peralte en la carretera.

- Ley de la Gravitación Universal:

- Concepto

- Leyes de Keppler:

- Primera Ley de Keppler

- Segunda Ley de Keppler

- Tercera Ley de Keppler

- Movimiento de los planetas 
- Movimiento de los satéites en órbitas circulares

- Fuerzas que actúan sobre los cuerpos:

- Fuerza de contacto

- Fuerza normal

- Fuerza de fricción:

- Estática

- Cinética

- Análisis de la manifestación de la energía cinética en la naturaleza.

- Diferencia entre fuerzas conservativas y disipativas, en base a las coordenadas de posición.

- Procesos para producir energía eléctrica por medio de la energía eólica, hidráulica y las mareas

- Fuerzas fundamentales de la naturaleza (nociones básicas):

- Gravitacional

- Magnética

- Eléctrica

- Electromagnética

- Leyes de Newton del movimiento:

- Primera Ley de Newton

- Segunda Ley de Newton

- Tercera Ley de Newton

- Diagramas de cuerpo libre en:

- Superficies horizontales

- Superficies inclinadas

- Cuerpos suspendidos

- Trabajo

- Concepto

- Fuerzas disipativas y fuerzas conservativas

- Teorema trabajo-energía

- Fluidos:

- Concepto

- Densidad:

- Concepto

- Principio de Pascal:

- Presión

- Presión en el Interior de un fluido

- Principio de Arquímedes:

- Fuerza de empuje

- Aplicaciones en máquinas hidráulicas

- Presión atmosférica:

- Definición

- Instrumentos de medida

- Dependencia de la altura

- Ley de Boyle.

- Relación entre presión, volumen y temperatura.

- Estudio del gas, y la presión que este ejerce

- Entropía:

- Primera y Segunda Ley de la Termodinámica

- Movimiento ondulatorio: 
- Concepto

- Tipos de ondas:

- Electromagnéticas

- Mecánicas:

- Transversales; Longitudinales

- Velocidad de propagación; Frecuencia; Periodo; Amplitud; Longitud de onda

- Fase; Reflexión; Refracción.

5.4.2 Propuesta de reestructuración para el programa de Física de 11‥

\section{Propuesta $11^{\circ}$ año}

- Carga eléctrica:

- Unidad de medida del S.I.

- Corriente continua

- Corriente alterna

- Cuerpos electrizados.

- Inducción y polarización.

- Ley de Coulomb para cargas puntuales.

- Campo eléctrico en una carga puntual:

- Unidad de medida del S.I.

- Líneas de fuerza

- Materiales:

- Conductores

- Dieléctricos

- Semiconductores y

- Superconductores

- Diferencia de potencial eléctrico:

- Unidad de medida

- Resistencia eléctrica:

- Ley de Ohm

- Circuitos en serie

- Circuitos en paralelo.

- Magnetismo:

- Imanes

- Campo magnético:

- Unidad de medida en el S.I.

- Fuerzas magnéticas sobre cargas móviles

- Vector campo magnético

- Aplicaciones en bobinas y solenoides

- Fuerza magnética.

- Electromagnetismo:

- El experimento de Oersted

- Funcionamiento del transformador eléctrico.

- Reflexión:

- Leyes de la reflexión: 
- En superficies pulidas

- En superficies rugosas

- Refracción de la luz:

- Velocidad de la luz en diferentes medios

- Ley de Snell

- Reflexión interna total.

- Descomposición de la luz visible:

- El arco iris

- Imágenes en espejos planos y espejos curvos:

- Imágenes reales; Imágenes virtuales

- Imágenes formadas en lentes convergentes y lentes divergentes.

- Naturaleza de la luz:

- Luz visible; Fuentes de luz

- Ley de la lluminación.

- Unidad de medida en el S.I.

- Intensidad luminosa.

- Unidades de medida de la intensidad luminosa, flujo luminoso e iluminación.

- Ley de la iluminación

- Teoría de la Relatividad: especial y general.

- Ideas de espacio, tiempo y masa a la luz de la nueva teoría.

- Ecuaciones del transformación del espacio", la masa y el tiempo.

- Equivalencia entre la masa y la energía de acuerdo a la teoría especial de la relatividad.

- Comportamiento dual de la luz.

- Carácter "dual" y "ondulatorio" de la luz.

- Concepto de cuanto de luz o "fotón".

- Similitudes y diferencias entre la explicación de Bohr y de De Broglie para las orbitas del átomo de Hidrógeno.

- El comportamiento de los planos cristalinos el la obtención de interferencias.

- Importancia de los experimentos de difracción para confirmar la hipótesis de pe Broglie sobre el carácter copular y ondulatorio de los electrones.

\subsubsection{Propuesta de reestructuración para el programa de Química de 10․}

\section{Propuesta $10^{\circ}$ año}

- Evolución histórica del desarrollo de la Química.

- Breve referencia de otros aportes de los diferentes científicos al modelo atómico: Plank, De Broglie y Heisenberg.

- Beneficios y peligros de la energía nuclear ${ }^{1}$.

- Números cuánticos y su relación con la estructura electrónica.

- Orbitales atómicos.

- Principio de exclusión de Pauli. y Regla de Hund.

- Configuraciones electrónicas: sistema $\mathrm{nl}^{\mathrm{x}}$, y diagrama orbital (flechas).

- Electrón diferenciante.

Electrones de valencia. 
- Elementos que presentan anomalías en su configuración electrónica.

- Tabla Periódica de los Elementos.

- Desarrollo histórico.

- Clasificación de los elementos: Representativos, Transición, Lantánidos y Actínidos.

- Relación de la estructura electrónica con la posición del elemento en la Tabla.

Periódica y la familia a la que pertenece.

- Números de oxidación de elementos representativos. Ubicación del último electrón (diferenciante).

- Elementos esenciales en los organismos vivos (oligoelementos) ${ }^{2}$.

- Ley Periódica. Propiedades Periódicas

- El enlace químico.

Formación de compuestos y las leyes que los rigen.

- Fórmula molecular, estructural y empírica.

- Teorías de enlace.

- Estructuras de Lewis de elementos representativos.

- Enlace iónico:

Características, propiedades de los compuestos iónicos.

- Enlace covalente: Formación y características

- Estructuras de Lewis para compuestos sencillos.

- Enlace covalente: sencillo, doble, triple y coordinado.

- Polar, no polar.

Propiedades de los compuestos moleculares.

- Teoría de la repuls ión de los pares de electrones de la capa de valencia (TRPECV).

- Polaridad de las moléculas.

- Teoría de enlace de valencia (hibridación de orbitales).

- Enlaces sigma y pi.

- Fuerzas intermoleculares:

Puente de hidrógeno, ion-dipolo, Dipolo - dipolo, Fuerzas de dispersión.

- Fuerzas iónicas.

- El enlace metálico. Teoría del mar de electrones.

- Clasificación de compuestos:

- Según el tipo de elementos presentes: ácidos (oxácidos), bases (hidróxidos), sales ternarias y cuaternarias.

- Uso y manejo de compuestos quím icos (ternarios y cuaternarios).

- Nomenclatura.

-Sistema Stoke para ácidos (oxácidos), bases (hidróxidos), sales ternarias y cuaternarias.

-Sistema estequiométrico para compuestos covalentes no metálicos.

- Cambios físicos y químicos.

- Contaminación por reacciones Químicas.

- Clasificación de reacciones Químicas:

- Combinación, descomposición, desplazamiento y doble descompos ición. 
- Combustión, ácido-base o neutralización, precipitación y oxidación - reducción.

- Reacciones Químicas de interés ambiental.

- Estequiometría:

-Cantidad de sustancia (mole), número de Avogadro, masa molar.

- Relaciones de moles, gramos, y número de partículas entre reactivos y productos.

Polímeros. Aplicaciones

- Biomateriales. Aplicaciones².

- Química moderna3:

Ejm: Nanotubos de carbono, u otros temas que el profesor considere relevantes.

1 y 2. Se propone desarrollar como trabajo extraclase.

1, 2, 3. Se propone desarrollarlos como trabajos de investigación o como trabajos grupales por medio del análisis de lecturas.

5.4.4 Propuesta de reestructuración para el programa de Química de 11ํ.

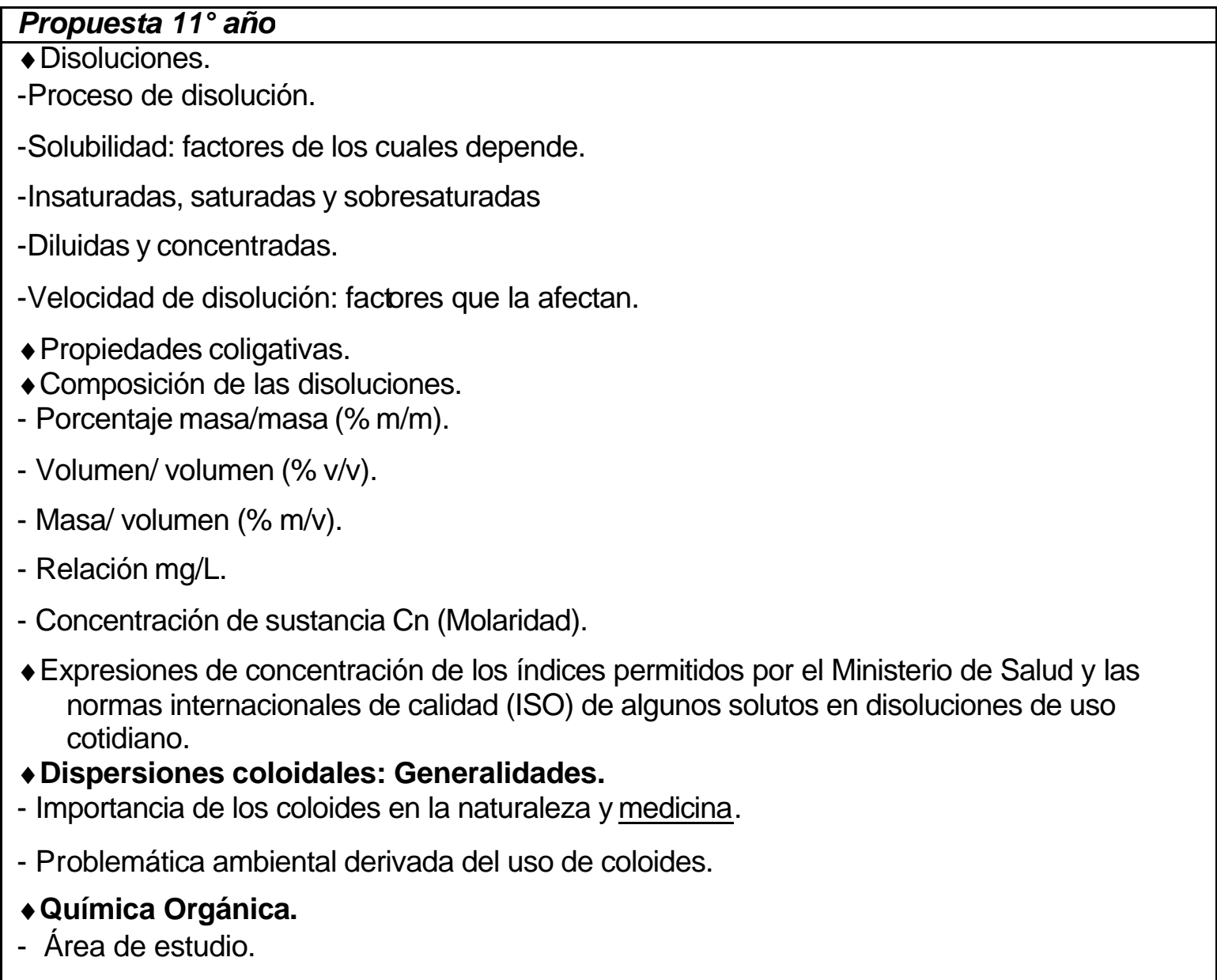




\section{- Importancia para la humanidad.}

\section{- El átomo de carbono.}

Propiedades: Hibridación de orbitales, geometría molecular, enlaces sigma y pi, homocombinación, tetravalencia, formas alotrópicas y anfoterismo.

- Enlace.

- Compuestos del carbono.

- Elementos que los constituyen.

- Estados de agregación en que se presentan.

- Compuestos Orgánicos en la conformación de los seres vivos.

- Hidrocarburos alifáticos:

- Clasificación: saturados e insaturados.

- Alcanos, alquenos y alquinos.

- Nomenclatura I.U.P.A.C.

- Hidrocarburos aromáticos.

- Impacto ambiental de los hidrocarburos.

- Grupos funcionales:

- Haluros, hidroxilo, carbonilo, carboxilo, amidas, aminas.

- Compuestos que presentan los grupos funcionales: haluros, alcoholes, aldehídos, cetonas, ácidos carboxílicos, aminas, amidas, aminoácidos.

- Biomoléculas

- Carbohidratos

- Lípidos

- Proteínas

- Aplicación de compuestos orgánicos.

La propuesta anterior se realizó con el fin de:

- Que los y las docentes dispongan de mayor tiempo para desarrollar los contenidos incluidos en la misma, debido a que se eliminan temas que también se desarrollan en niveles inferiores, evitando así la repetición de contenidos.

- Que el proceso de aprendizaje en el aula pueda llevarse a un ritmo en el cual el educando pueda disponer de mayor tiempo para comprender de manera significativa los contenidos.

- Que se puedan abarcar temas de actualidad científica y tecnológica, que no están incluidos en los programas de estudio y que sean de interés tanto del docente como de los alumnos. 


\section{REFERENCIAS}

Brown, Theodore; LeMay, Eugene; Bursten, Buce y Burdge, Jlia. (2004). Química. La ciencia central. México D.F., México: Prentice Hall.

Bunge, Mario. (1984). Ciencia y desarrollo. Buenos Aires, Argentina: Ediciones siglo veinte.

Darrel, Barnald J. (1970). Enseñanza de las ciencias. Buenos Aires, Argentina: Librería del colegio.

Guardián, Alicia. (1999). Política Social y Educación en Costa Rica (comp.). San José, Costa Rica: UNICEF.

Hernández, Roberto; Fernández, Carlos y Baptista, Pilar. (2006). Metodología de la investigación. México D.F., México: McGRAW-HILL.

Herrera, Leonardo y Artavia, Yeudin. (2008). Evolución de los programas de enseñanza media en el área de Física y de Química propuestos por el Ministerio de Educación Pública, para el ciclo diversificado. Período 1980-2005. Trabajo final de graduación para optar por el grado de Licenciados en enseñanza de la Física y la química, Escuela de Formación Docente, Universidad de Costa Rica, San José, Costa Rica.

Martínez, Eduardo. (1994). Ciencia, tecnología y desarrollo: interrelaciones teóricas y metodológicas. Caracas, Venezuela: Nueva Sociedad.

Morales, Orlando. (2001). Ciencia, tecnología y técnicas -ensayos-. San José, Costa Rica: INA.

Ministerio de Educación Pública - Costa Rica. (1980). Política educativa para 1980. San José, Costa Rica.

Ministerio de Educación Pública - Costa Rica. (1994). Política educativa hacia el siglo XXI. San José, Costa Rica.

Ministerio de Educación Pública - Costa Rica. (1980). Programas de estudio de Física. Educación Diversificada. San José, Costa Rica.

Ministerio de Educación Pública - Costa Rica. (1991). Programas de estudio de Física. Educación Diversificada. San José, Costa Rica.

Ministerio de Educación Pública - Costa Rica. (1996). Programas de estudio de Física. Educación Diversificada. San José, Costa Rica.

Ministerio de Educación Pública - Costa Rica. (2001). Programas de estudio de Física. Educación Diversificada. San José, Costa Rica.

Ministerio de Educación Pública - Costa Rica. (2005). Programas de estudio de Física. Educación Diversificada. San José, Costa Rica. 
Ministerio de Educación Pública - Costa Rica. (1980). Programas de estudio de Química. Educación Diversificada. San José, Costa Rica.

Ministerio de Educación Pública - Costa Rica. (1991). Programas de estudio de Química. Educación Diversificada. San José, Costa Rica.

Ministerio de Educación Pública - Costa Rica. (1996). Programas de estudio de Química. Educación Diversificada. San José, Costa Rica.

Ministerio de Educación Pública - Costa Rica. (2001). Programas de estudio de Química. Educación Diversificada. San José, Costa Rica.

Ministerio de Educación Pública - Costa Rica. (2005). Programas de estudio de Química. Educación Diversificada. San José, Costa Rica.

Pozo, Juan Ignacio y Gómez, Mguel Ángel. (1998). Aprender y enseñar ciencia. Del conocimiento cotidiano al conocimiento científico. Madrid, España: EDICIONES MORATA. S.L.

UNICEF (1970). Nuevas tendencias en la enseñanza de las ciencias. UNESCO, Santiago de Chile.

Wilson, Jerry y Buffa, Anthony. (2003). Física (5ª ed.). México D.F., México: Prentice Hall. 